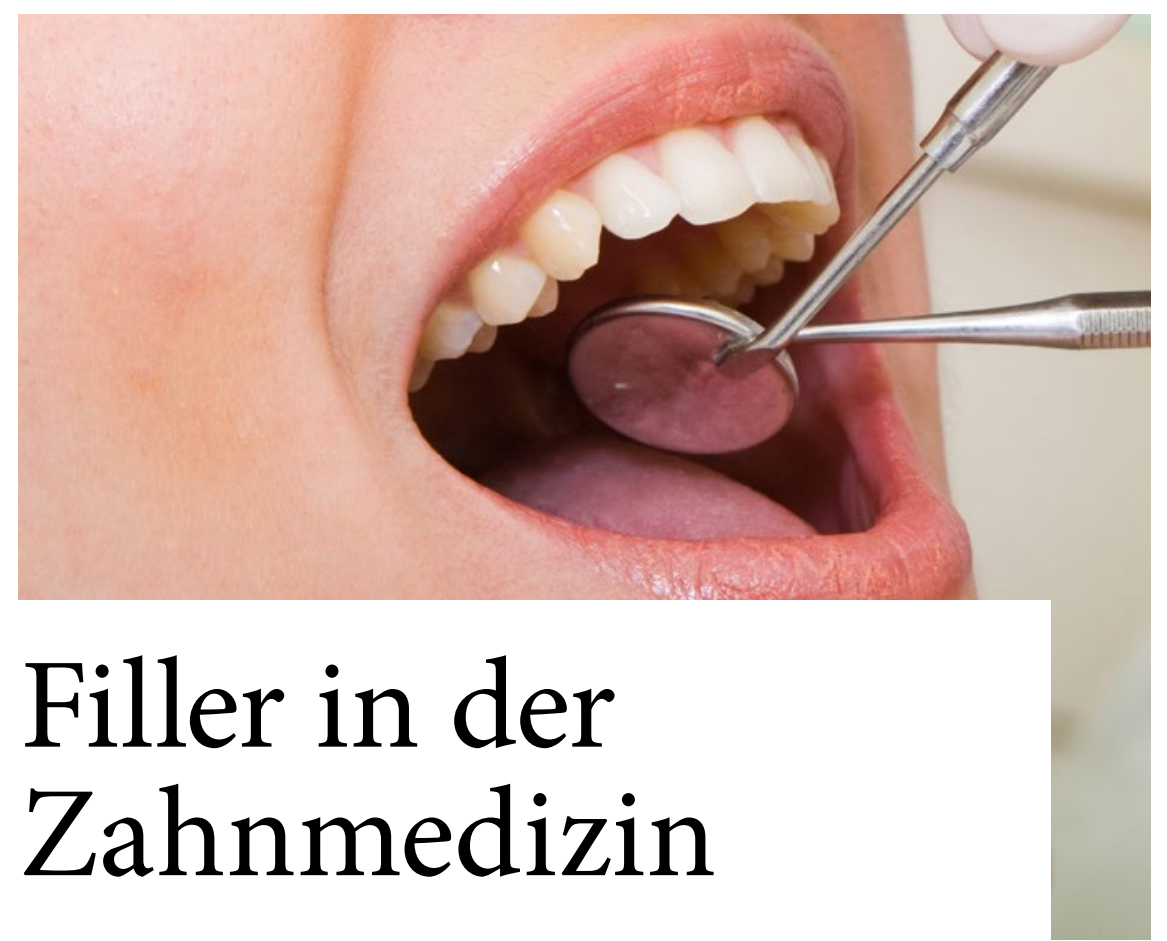

Hyaluronsäure zur Rekonstruktion der Interdentalpapille. In der heutigen Zahnheilkunde spielt neben der Wiederherstellung der Kaufunktion die Zahn- und Gingivaästhetik eine immer wichtigere Rolle. Insbesondere im Bereich der oberen Schneidezähne sind die Girlandenform der Gingiva und die Ausbildung der Interdentalpapillen von herausragender Bedeutung.

AUTOR: PROF. DR. DR. FELIX KOCH

Neben der Ästhetik vermeidet eine intakte Inderdentalpapille auch die Einlagerung von Speisen zwischen den Zähnen und beeinflusst die Phonetik. Zur Herstellung einer intakten Papille sollten der interdentale Kontaktpunkt und der crestale Knochen den Abstand von fünf Millimetern nicht überschreiten. Eine weitere wichtige Größe ist der approximale Abstand der Zahnwurzeln, der einen Abstand von vier bis sechs Millimetern nicht überschreiten sollte. Chen et al. untersuchten die Inderdentalpapillen der Oberkieferfront: Bei einem Abstand von unter vier Millimetern war die Papille immer vorhanden, bei einem Abstand von über sieben Millimetern hingegen nie.

Chirurgische Maßnahmen zur Wiederherstellung der Interdentalpapille bedie- nen sich unterschiedlicher Lappentechniken. Dabei sind die Ergebnisstabilität und Vorhersagbarkeit zweifelhaft. Neben der plastischen Parodontalchirurgie kommen daher auch restaurative, prothetische und kieferorthopädische Verfahren zum Einsatz. Becker et al. publizierten als Erste die Möglichkeit, Hyaluronsäure zum Aufbau der Zahnpapille zu nutzen.

\section{ERFOLGREICHE REKONSTRUKTION}

In einer Studie von Lee et al. 2016 wurden die Interdentalpapillen mithilfe einer 30 G-Kanüle per Hyaluronsäure aufgespritzt. Die Kanüle wurde in einem Winkel von 45 Grad zwei bis drei Millimeter apikal der involvierten Papille inseriert. Insgesamt fünfmal wurden jeweils $0,002 \mathrm{cc}$ appliziert, damit schließlich ein Volumen von 0,01 cc eingebracht worden war. Diese

\section{Wissenschaftlicher Info-Dienst}

Der WID bringt alle zwei Monate Abstracts von wissenschaftlichen Publikationen und Vorträgen mit hohem Praxisbezug.

\section{$\triangle$ Für ein Jahresabo zahlen FVDZ-Mitglieder 33 Euro (Nichtmitglieder 49 Euro).}
$\triangle$ Studentische FVDZ-Mitglieder können den WID für 15 Euro pro Jahr abonnieren.

\section{$\triangle$ Informationen gibt es in der FVDZ-Bundesgeschäftsstelle bei Eva Schaefer unter der Telefonnummer 0228 - 855730, weitere Infos unter www.fvdz.de.}

Prozedur wiederholte sich fünfmal in einem Abstand von drei Wochen, bis die schwarzen Interdentalpapillen klinisch nicht mehr sichtbar waren. Die Auswertung erfolgte sechs Monate nach dem Eingriff: 36 der 57 Papillen erreichten eine komplette Rekonstruktion, wobei 37 Prozent eine partielle Rekonstruktion von 19 bis 96 Prozent zeigten. Becker et al. erreichten eine komplette Papillenrekonstruktion in 21 Prozent der Fälle, bei den übrigen wurden 57 bis 97 Prozent rekonstruiert. Mansouri et al. berichten in 22 bis 100 Prozent von einer interdentalen Papillenrekonstruktion, wobei beide nur drei Injektionen verabreichten.

Die Studie von Lee et al. korrelierte zusätzlich den Abstand des crestalen Knochens zum interdentalen Kontaktpunkt. Dabei zeigte sich dessen maßgeblicher Einfluss auf die erfolgreiche Rekonstruktion der Interdentalpapille.

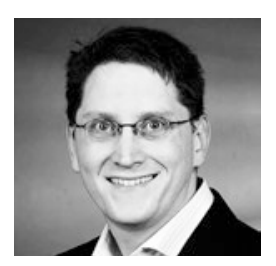

Prof. Dr. Dr. Felix P. Koch Autor des Wissenschaftlichen InfoDienstes, Lehrbeauftragter der Uniklinik Frankfurt, Praxisklinik für Mund-, Gesichtschirurgie in Wiesbaden E-Mail: wid@fvdz.de 